In Cres. Vol. $4 N^{\circ} 1:$ pp. 51-62, 2013

\title{
ESTILO DE VIDA Y FACTORES BIOSOCIOCULTURALES DE LOS DOCENTES DE LA INSTITUCIÓN EDUCATIVA REPÚBLICA DE CHILE - CASMA, 2012*
}

\author{
LIFE STYLE AND BIOSOCIOCULTURAL FACTORS OF THE TEACHERS \\ OF REPUBLIC OF CHILE EDUCATIVE INSTITUTION - CASMA, 2012
}

\author{
Kirenia Enghel Gonzales Zegarra \\ Yolanda Rodriguez Núñez ${ }^{2}$
}

\section{RESUMEN}

El presente trabajo de investigación fue cuantitativo, de corte transversal y diseño descriptivo correlacional. El objetivo fue determinar la relación entre el estilo de vida y los factores biosocioculturales de los docentes de la Institución Educativa República de Chile, Casma (Perú), 2012. El universo muestral estuvo constituido por 53 docentes; se utilizaron dos instrumentos para la recolección de datos: 1) La escala del estilo de vida y 2) el cuestionario sobre factores biosocioculturales, a través de la técnica de la entrevista. Los datos fueron procesados con el Software SPSS, versión 18.0. Para establecer la relación entre las variables de estudio se aplicó la prueba de independencia de criterios Chi cuadrado, con el 95\% de confiabilidad y significancia de $\mathrm{p}<0,05$. Más de la mitad de los docentes tuvieron un estilo de vida saludable y menos de la mitad, no saludable. En relación a los factores biosocioculturales, la mayoría fueron adultos maduros; más de la mitad, de sexo masculino. En cuanto a religión, la mayoría fueron católicos, con un ingreso económico mayor de 1000 nuevos soles; y de estado civil, poco menos de la mitad, casados. No se encontró relación significativa entre el estilo de vida y los factores biosocioculturales.

PALABRAS CLAVE: Estilo vida, factores biosocioculturales, adulto.

* Recibido: 30 de marzo del 2013; aprobado: 16 de abril del 2013.

1 Alumna del VIII ciclo de la escuela profesional de Enfermería de la Universidad Católica Los Ángeles de Chimbote.

2 Doctora en Enfermería, Docente Principal de la Escuela de Enfermería de la ULADECH Católica. Jefa de Sección de Investigación en Enfermería . 


\begin{abstract}
The present research was quantitative, cross-sectional and descriptive correlational design. The goal was to determine the relationship between lifestyle factors and teachers Biosocioculturales of I.E. Republic of Chile, Casma, 2012. The sample universe consisted of 53 teachers, two instruments were used to collect data: The scale of lifestyle factors and questionnaire biosocioculturales through interview technique. The data were processed with SPSS software version 18.0. To establish the relationship between the variables of study applied the test of independence of criteria Chi square, with $95 \%$ reliability and significance of $p<0,05$. Concluding that: More than half of teachers have a healthy lifestyle and less than half have a lifestyle unhealthy. In relation to the factors that most biosocioculturales we are mature adults, more than half are male, in religion most are Catholics, income greater than 1000 new soles and marital little less than half married. There is no statistically significant relationship between lifestyle factors and teacher biosocioculturales in I.E. Republic of Chile, Casma.
\end{abstract}

KEY WORDS: Lifestyle, biosocioculturales factors, adult.

\title{
INTRODUCCIÓN
}

La preservación de la salud física y mental constituye una responsabilidad individual, con independencia de las acciones dirigidas a su conservación en el ciudadano y representa un deber cívico para evitar añadir una carga económica o sentimental a corto o largo plazo, a la familia o a la propia sociedad, dando la posibilidad de alcanzar una vejez sana, portadora de ricas y ejemplarizantes experiencias para los más jóvenes. Tal legado es un estilo de vida. El estilo de vida es entendido como los hábitos de vida, la forma de vida, el conjunto de comportamientos o las actitudes que desarrollan las personas que unas veces son saludables y, otras, nocivas para la salud. ${ }^{1}$

En los países subdesarrollados, los estilos de vida poco saludables son los que causan la mayoría de enfermedades. El estilo de vida que tiene la persona en sus años previos, si nunca hizo actividad física, su nutrición fue deficiente o tuvo hábitos nocivos, tendrá problemas en su vejez; de ahí la importancia de las actividades y programas de promoción y prevención en la salud.

Para disminuir la incidencia y mortalidad por enfermedades prevenibles es necesario identificar las circunstancias que favorecen el desarrollo y la aceleración de las enfermedades, siendo importante el intento por controlar este problema, valorar la presencia de riesgos modificables o controlables para, luego, actuar en ello, considerando ajustes en los estilos de vida de las personas.

Muchos de los estilos de vida incluyen fundamentalmente una alimentación adecuada, promoción de la actividad física, control del tabaquismo y de 
otras adicciones, ambientes saludables en viviendas y trabajos, conductas sexuales, salud mental y control de tres elementos ligados a la alimentación: presión arterial, colesterol y arteriosclerosis. ${ }^{3}$

A esta realidad, no escapa la población de docentes de la Institución Educativa (I.E.) República de Chile, del distrito de Casma, provincia de Casma, departamento de Ancash. Dicha población de docentes es de 53. Tienen diversas edades, algunos laborando más de 35 años en la misma I.E. La mayoría procede del distrito de Casma. ${ }^{4}$

\section{PROBLEMA}

¿Existe relación entre el estilo de vida y los factores biosocioculturales de los docentes de la I.E. República de Chile - Casma, 2012?

\section{OBJETIVO GENERAL}

Determinar la relación entre el estilo de vida y los factores biosocioculturales de los docentes de la I.E. República de Chile, Casma.

\section{OBJETIVOS ESPECÍFICOS}

1. Valorar el estilo de vida de los docentes de la I.E. República de Chile, Casma.

2. Identificar los factores biosocioculturales: sexo, religión, estado civil e ingreso económico de los docentes de la I.E. República de Chile, Casma.

\section{HIPÓTESIS}

Existe relación significativa entre el estilo de vida y los factores biosocioculturales de los docentes de I.E. República de Chile, Casma.

\section{METODOLOGÍA}

El presente estudio fue de tipo cuantitativo, de corte transversal y diseño descriptivo correlacional. El universo muestral estuvo constituido por 53 docentes. Se utilizaron los siguientes criterios de inclusión: docente que labore en la I. E. República de Chile; docente que acepta participar en el estudio; docente de ambos sexos. ${ }^{5-8}$

En la recolección de datos, se aplicó la técnica de la entrevista, utilizándose dos instrumentos: la escala del estilo de vida elaborado por Walker, Sechrist 
y Pender, y modificado por Delgado R; Reyna E y Díaz R, y el cuestionario semiestructurado sobre factores biosocioculturales elaborado por Delgado R; Reyna E y Díaz R.

Para el procesamiento y análisis estadístico se utilizó el software especializado de estadística SPSS versión 18.0, empleando tablas simples y de doble entrada para la presentación de resultados. Para establecer la relación entre las variables de estudio se aplicó la prueba de independencia de criterios Chi cuadrado, con el $95 \%$ de confiabilidad y significancia de $\mathrm{p}<0,05$.

\section{RESULTADOS}

Tabla 01

ESTILO DE VIDA DE LOS DOCENTES DE LA

I.E. REPÚBLICA DE CHILE, CASMA, 2012

\begin{tabular}{lcc}
\hline Estilo de vida & Frecuencia & Porcentaje \\
\hline Saludable & 29 & 54,7 \\
No saludable & 24 & 45,3 \\
\hline Total & 53 & 100,0 \\
\hline $\begin{array}{l}\text { Fuente: Escala del estilo de vida elaborada por: Walker, Sechrist y Pender } \\
\text { modificado por: Díaz, R; Reyna, E; Delgado, R. aplicado a los docentes de la I.E. } \\
\text { República de Chile, Junio 2012. }\end{array}$
\end{tabular}

Tabla 02

FACTORES BIOSOCIOCULTURALES DE LOS DOCENTES

\begin{tabular}{lrr}
\hline \multicolumn{3}{c}{ FACTORES BIOLÓGICOS } \\
\hline Edad (años) & $\mathbf{n}$ & $\%$ \\
20 a 35 & 6 & 11,3 \\
36 a 59 & 43 & 81,1 \\
60 a más & 4 & 7,5 \\
Total & 53 & 100,0 \\
Sexo & $\mathbf{n}$ & $\%$ \\
Masculino & 29 & 54,7 \\
Femenino & 24 & 45,3 \\
Total & 53 & 100,0 \\
\hline & $\mathbf{n}$ & $\mathbf{\%}$ \\
\hline Religión & FACTORES CULTURALES & 79,2 \\
Católica & 52 & 9,5 \\
Evangélica & 5 & 11,3 \\
Otras & 6 & 100,0 \\
Total & 53 & \\
\hline
\end{tabular}




\begin{tabular}{lrr}
\hline \multicolumn{3}{c}{ FACTORES SOCIALES } \\
\hline Estado civil & $\mathbf{n}$ & $\%$ \\
Soltero(a) & 20 & 37,7 \\
Casado(a) & 24 & 45,3 \\
Viudo(a) & 2 & 3,8 \\
Unión libre & 6 & 11,3 \\
Separado(a) & 1 & 1,9 \\
Total & 53 & 100,0 \\
Ingreso económico (soles) & $\mathbf{n}$ & $\mathbf{\%}$ \\
Menos de 100 & 1 & 1,9 \\
De 100 a 599 & 2 & 3,8 \\
De 600 a 1000 & 12 & 22,6 \\
Más de 1000 & 38 & 71,7 \\
Total & 53 & 100,0 \\
\hline Fuente: Cuestionario de factores biosoculturales, elaborado por: Díaz, R; Reyna, \\
E; Delgado, R (2008). Aplicado a los docentes de la I.E. República de Chile, Junio \\
2012.
\end{tabular}

Tabla 03

FACTORES BIOLÓGICOS Y ESTILO DE VIDA DE LOS DOCENTES

\begin{tabular}{lrrrrrr}
\hline \multirow{2}{*}{$\begin{array}{l}\text { Edad } \\
\text { (años) }\end{array}$} & \multicolumn{9}{c}{ Estilo de vida } \\
& \multicolumn{2}{c}{ Saludable } & \multicolumn{2}{c}{ No saludable } & \multicolumn{2}{c}{ Total } \\
\cline { 2 - 8 } & \multicolumn{1}{c}{ n } & $\%$ & n & $\%$ & n & $\%$ \\
\hline $20-35$ & 4 & 7,5 & 2 & 3,8 & 6 & 11,3 \\
$36-59$ & 22 & 41,5 & 21 & 39,6 & 43 & 81,1 \\
60 a más & 3 & 5,7 & 1 & 1,9 & 4 & 7,5 \\
Total & 29 & 54,7 & 24 & 45,3 & 53 & 100,0 \\
\hline
\end{tabular}

$$
\begin{gathered}
\text { Chi cuadrado } \\
\mathrm{X}^{2}=1,229,2 \mathrm{gl} \\
\mathrm{P}=0,541>0,05
\end{gathered}
$$

No existe relación entre las variables. 
Tabla 03 (Cont.)

\begin{tabular}{|c|c|c|c|c|c|c|}
\hline \multirow{3}{*}{ Sexo } & \multicolumn{6}{|c|}{ Estilo de vida } \\
\hline & \multicolumn{2}{|c|}{ Saludable } & \multicolumn{2}{|c|}{ No saludable } & \multicolumn{2}{|c|}{ Total } \\
\hline & $\mathrm{n}$ & $\%$ & $\mathbf{n}$ & $\%$ & $\mathbf{n}$ & $\%$ \\
\hline Masculino & 16 & 30,2 & 13 & 24,5 & 29 & 54,7 \\
\hline Femenino & 13 & 24,5 & 11 & 20,8 & 24 & 45,3 \\
\hline Total & 29 & 54,7 & 24 & 45,3 & 53 & 100,0 \\
\hline \multicolumn{7}{|c|}{ Prueba del Chi cuadrado } \\
\hline \multicolumn{7}{|c|}{$\mathrm{X}^{2}=0,005,1 \mathrm{gl}$} \\
\hline \multicolumn{7}{|c|}{$\mathrm{P}=0,942>0,05$} \\
\hline \multicolumn{7}{|c|}{ No existe relación entre las variables. } \\
\hline
\end{tabular}

Fuente: Cuestionario de factores biosoculturales, elaborado por: Díaz, R; Reyna, E; Delgado, R y Escala del estilo de vida elaborada por: Walker, Sechrist y pender, modificado por: Díaz, R; Reyna, E; Delgado, R. Aplicado a los docentes de la I.E. República de Chile, Junio 2012.

Tabla 04

FACTORES CULTURALES Y ESTILO DE VIDA DE LOS DOCENTES

\begin{tabular}{lrrrrrr}
\hline \multirow{2}{*}{ Religión } & \multicolumn{9}{c}{ Estilo de vida } \\
\cline { 2 - 8 } & \multicolumn{2}{c}{ Saludable } & \multicolumn{2}{c}{ No saludable } & \multicolumn{2}{c}{ Total } \\
\cline { 2 - 8 } & $\mathrm{n}$ & $\boldsymbol{\%}$ & $\mathbf{n}$ & $\boldsymbol{\%}$ & $\mathbf{n}$ & $\%$ \\
\hline Católico & 22 & 41,5 & 20 & 37,7 & 42 & 79,2 \\
Evangélico(a) & 2 & 3,8 & 3 & 5,7 & 5 & 9,5 \\
Otras & 5 & 9,4 & 1 & 1,9 & 6 & 11,3 \\
Total & 29 & 54,7 & 24 & 45,3 & 53 & 100,0 \\
\hline
\end{tabular}

Prueba del Chi cuadrado

$$
\begin{gathered}
\mathrm{X}^{2}=2,513,2 \mathrm{gl} \\
\mathrm{P}=0,285>0,05
\end{gathered}
$$

No existe relación entre las variables.

Fuente: Cuestionario de factores biosoculturales, elaborado por: Díaz, R; Reyna, E; Delgado, R y Escala del estilo de vida elaborada por: Walker, Sechrist y pender, modificado por: Díaz, R; Reyna, E; Delgado, R. Aplicado a los docentes de la I.E. República de Chile, Junio 2012. 
Tabla 05

FACTORES SOCIALES Y ESTILO DE VIDA DE LOS DOCENTES

\begin{tabular}{lrrrrrr}
\hline & \multicolumn{9}{c}{ Estilo de vida } \\
\cline { 2 - 8 } Estado civil & \multicolumn{2}{c}{ Saludable } & \multicolumn{2}{c}{ No saludable } & \multicolumn{2}{c}{ Total } \\
\cline { 2 - 8 } & $\mathrm{n}$ & $\mathbf{\%}$ & $\mathbf{n}$ & $\mathbf{0}$ & $\mathbf{n}$ & $\mathbf{\%}$ \\
\hline Soltero(a) & 9 & 17,0 & 11 & 20,8 & 20 & 11,3 \\
Casado(a) & $\mathbf{1 6}$ & $\mathbf{3 0 , 2}$ & $\mathbf{8}$ & $\mathbf{1 5 , 1}$ & $\mathbf{2 4}$ & $\mathbf{4 5 , 3}$ \\
Viudo(a) & 2 & 3,8 & 0 & 0,0 & 2 & 7,5 \\
Unión libre & 2 & 3,8 & 4 & 7,5 & 6 & 11,3 \\
Separado(a) & 0 & 0,0 & 1 & 1,9 & 1 & 1,9 \\
Total & 29 & 54,7 & 24 & 45,3 & 53 & 100,0 \\
\hline
\end{tabular}

Prueba del Chi cuadrado

$$
\begin{gathered}
\mathrm{X}^{2}=6,116 ; 4 \mathrm{gl} \\
\mathrm{P}=0,191>0,05
\end{gathered}
$$

No existe relación entre las variables.

\begin{tabular}{lrrrrrr}
\hline \multirow{2}{*}{$\begin{array}{c}\text { Ingresos } \\
\text { económicos } \\
\text { (Nuevos Soles) }\end{array}$} & \multicolumn{9}{c}{ Estilo de vida } \\
\cline { 2 - 8 } & \multicolumn{2}{c}{ Saludable } & \multicolumn{2}{c}{ No saludable } & \multicolumn{2}{c}{ Total } \\
\hline Menos de 100 & 0 & 0,0 & 1 & 1,9 & 1 & 1,9 \\
$100-599$ & 1 & 1,9 & 1 & 1,9 & 2 & 3,8 \\
$600-1000$ & 6 & 11,3 & 6 & 11,3 & 12 & 22,6 \\
Más de 1000 & 22 & 41,5 & 16 & 30,2 & 38 & 71,7 \\
Total & 29 & 54,7 & 24 & 45,3 & 53 & 100,0 \\
\hline
\end{tabular}

Prueba del Chi cuadrado

$$
\begin{gathered}
\mathrm{X}^{2}=1,489 ; 3 \mathrm{gl} \\
\mathrm{P}=0,685>0,05
\end{gathered}
$$

No existe relación entre las variables.

Fuente: Cuestionario de factores biosoculturales, elaborado por: Díaz, R; Reyna, E; Delgado, R y Escala del estilo de vida elaborada por: Walker, Sechrist y pender, modificado por: Díaz, R; Reyna, E; Delgado, R.

Aplicado a los docentes de la I.E. República de Chile, Junio 2012. 


\section{DISCUSIÓN}

Tabla 01: Los resultados evidencian que más de la mitad de los docentes que laboran en la I.E. República de Chile presentan un estilo de vida saludable, posiblemente debido a que su misma rutina de trabajo presenta exigencias como mantenerse activos la mayor parte del día, mantener la mente activa al generar continuamente nuevas estrategias para brindar educación y mantenerse físicamente sanos; así como también, otorgan importancia al tema de su salud, buscando acudir al médico para una consulta de por lo menos una vez al año para mantener su salud y no estar enfermos. También se puede atribuir a que todos se encuentran asegurados en Essalud, y que la mayoría recibe un sueldo mensual mayor de mil Nuevos Soles.

Los resultados empíricos se relacionan con el estudio de Mendoza, $\mathbf{M} .{ }^{9}$ sobre estilo de vida y factores biosocioculturales de la persona adulta del AA.HH Julio Meléndez, de Casma, donde encontró que el 63,3\% de adultos encuestados muestran un estilo de vida saludable y el $36.4 \%$, no saludable.

Tabla 02: Los resultados evidencian que el docente de la I.E. República de Chile, en relación al factor biológico demostraron que más de la mitad tiene edades entre 36-59 años, encontrándose en la etapa del adulto maduro, lo cual se puede deber probablemente a que en esta edad las personas ya han alcanzado ser profesionales. También se demostró que más de la mitad son del sexo masculino, debido a que los hombres tienen más oportunidades de estudiar y, por ende, trabajar para el sustento de su familia.

Con respecto al factor cultural, la mayoría son de religión católica, atribuyéndose a que la mayor parte de la ciudad, a la que pertenecen, es de esta misma religión; así mismo, puede ser que fue la religión que les transmitieron sus padres.

En los factores sociales, se encontró que la mayoría son casados, debido a que la edad predominante fue la del adulto maduro, viendo que es en esta edad cuando la mayoría de personas decide formar este vínculo; también puede ser porque la mayoría son católicos, poniendo énfasis el respeto de sus principios morales y religiosos o debido a su madurez física y mental, por lo cual, asumen esta responsabilidad. Así mismo, se encontró que el ingreso mensual de la mayoría es más de 1000 Nuevos Soles, lo que les asegura una estabilidad económica y una vida tranquila, para ellos y sus familias.

Estos resultados se aproximan a los datos obtenidos por Regalado, E; Osorio, $\mathrm{Y}^{10}$ en su estudio Estilo de vida y factores biosocioculturales de la persona adulta, Carhuaz, teniendo como resultado que el 51,4\% de las personas adultas sean de sexo masculino; y que el 83,6\%, sean de religión católica. 
De igual forma, se asemejan a los datos obtenidos por Felipe, $\mathrm{L}^{11}$ en su estudio Estilo de vida y factores biosocioculturales del adulto mayor en el AA. HH. Villa Magisterial I y II etapa, Nuevo Chimbote, teniendo como resultado que el $82 \%$ del adulto mayor sea de religión católica y que el $68 \%$, de estado civil casado.

Tabla 03: Al relacionar la variable edad con el estilo de vida utilizando el estadístico Chi-cuadrado de independencia $\left(\mathrm{X}^{2}=1,229 ; 2 \mathrm{gl}\right.$ y $\left.\mathrm{p}>0,05\right)$, se encontró que no existía relación significativa entre ambas variables; así mismo, la relación entre el sexo y estilo de vida, el Chi-cuadrado de independencia $\left(\mathrm{X}^{2}=0,005 ; 1 \mathrm{gl}\right.$ y $\left.\mathrm{p}>0,05\right)$, demostró que no existía relación significativa.

Esto quiere decir que la edad de los docentes no es un factor determinante para que tengan un estilo de vida saludable. Los docentes con un estilo de vida saludable, comprendidos en la etapa del adulto maduro, probablemente, tienen hábitos y costumbres ya formadas que difícilmente se pueden cambiar; los hábitos de buscar o interesarse por nuevos conocimientos dependen de la persona y de la motivación.

Entre la variable sexo y estilo de vida, no existe relación significativa. Esto quiere decir que el sexo no es un factor determinante para que los docentes tengan estilos de vida saludables. Los docentes que tienen estilo de vida saludable, de sexo masculino o femenino, probablemente por su misma rutina, duermen sus 8 horas diarias, se organizan para conseguir la solución de los problemas y se mantienen activos la mayor parte del día. Además, cuando tienen alguna molestia acuden al centro médico de salud.

Estos resultados se relacionan con el trabajo de Fernández, X. y Méndez, E. ${ }^{12}$ denominado Estilos de Vida y Factores Biológicos para la Salud de las Personas Adultas del Proyecto CRELLES, en donde las variables edad y estilo de vida no guardan relación significativa.

Sin embargo, los resultados contradicen a los encontrados por Fernández, L. y Reyes, S. ${ }^{13}$ quienes confirman que en las personas adultas se incrementan gradualmente la propensión a las incapacidades, enfermedades y a los accidentes, lo cual influye seriamente en el estado de bienestar; por lo tanto, el riesgo de enfermar se incrementa con la edad, en cualquier extracto socioeconómico.

Tabla 04: Al relacionar la religión y estilo de vida, con el Chi-cuadrado de independencia $\left(\mathrm{X}^{2}=2,513 ; 2 \mathrm{gl}\right.$ y $\left.\mathrm{p}>0,05\right)$ se encontró que no existía relación significativa entre ambos aspectos.

Este estudio se relaciona con el trabajo de Lazarte, C. y Méndez, A. ${ }^{14}$ titulado "Estilo de Vida y Factores Biosocioculturales de la Persona Adulta del 
AA.HH. Fraternidad, Chimbote", quienes hallaron que las variables estilo de vida y religión no tienen relación significativa.

Por otro lado, los resultados difieren a los obtenidos por Solis, R. y Chancahuaña V. ${ }^{15}$ en su estudio titulado Estilo de Vida y Factores Biosocioculturales de la Persona Adulta del centro poblado Huallcor, Huarazen, donde concluyeron que la religión tienen relación significativa con el estilo de vida.

En la variable religión y estilo de vida, no hay relación significativa. Esto quiere decir que la religión no es un factor determinante para que los docentes tengan un estilo de vida saludable. Esto puede deberse a que la religión católica trae consigo prácticas no saludables, por las costumbres como las numerosas festividades que se celebran y que implican muchas veces el consumo de alcohol, cigarrillo, comidas chatarras, gastos que afectan en la economía de la familia y que bien podrían ser aprovechadas en el mantenimiento de la salud.

Las creencias religiosas pueden ser buenas, no sólo para la salud espiritual sino también para los efectos positivos en el cuerpo. Con cierta regularidad, aparecen estudios que indican que la oración o la participación regular en servicios religiosos pueden ayudar a la salud de los creyentes. ${ }^{16}$

Tabla 05: Al relacionar el estado civil con el estilo de vida utilizando el Chi-cuadrado de independencia $\left(\mathrm{X}^{2}=6,116 ; 4 \mathrm{gl}\right.$ y $\left.\mathrm{p}>0,05\right)$ se encontró que no existía relación significativa entre ambas variables. El chi cuadrado también demostró que no hubo relación significativa entre el ingreso económico y el estilo de vida.

Se encontró semejanza con lo investigado por Cordero, M y Silva, $\mathbf{M}^{(17)}$ en su estudio de investigación denominado Estilo de Vida y Factores Biosocioculturales de la Persona Adulta Essalud, Casma, donde establecen que no existe relación significativa entre el estilo de vida y el estado civil e ingreso económico.

Los resultados del presente estudio contradicen a lo encontrado por Cruz, M. y Reyes, F. en su estudio "Estilo de Vida y factores Biosocioculturales de la persona adulta del centro poblado menor de Ichoca, Huaraz", ${ }^{18}$ quienes encontraron que sí existe relación significativa entre el estado civil y estilo de vida, concluyendo que el estado civil es un factor predominante para que los adultos tengan un estilo de vida definido.

$\mathrm{Al}$ analizar el estado civil y estilo de vida, de acuerdo a los hallazgos encontrados, se observa que no existe relación significativa entre ambas variables; es decir, los docentes, sin importar que sean casados o no, mantienen estilos de vida saludables, ya que generalmente traen costumbres de crianza en el 
hogar; así mismo, las practican con la familia que formarán; además, la forma de vivir para ellos no es un factor que dañe la integración familiar.

Por consiguiente, al analizar las variables ingreso económico y estilo de vida, de acuerdo a los resultados de esta investigación, se observa que no existe relación entre ambas variables; porque de una u otra manera el factor ingreso económico no va repercutir significativamente en que los docentes adopten estilos de vida saludables, siendo así que, teniendo un buen sueldo, comen comidas "chatarras", por ser lo que es más rápido de conseguir en el cafetín de su centro donde laboran o también porque no tienen tiempo para salir a recrearse por tener que cumplir con sus obligaciones laborales, así como también de no asistir a sus controles médicos.

\section{CONCLUSIONES}

La mayoría de los adultos tiene un estilo de vida saludable y un número significativo de ellos tienen estilo de vida no saludable.

En los factores biosocioculturales, la mayoría son adultos maduros, sexo masculino, religión católica y con ingresos económicos mayores de 1000 Nuevos Soles mensuales; menos de la mitad son solteros y casados.

El estilo de vida y los factores biosocioculturales, no tienen relación significativa.

\section{REFERENCIAS BIBLIOGRÁFICAS}

1 SCIELO - SCIENTIFIC EleCtronic LibRARy Online. CONCYTEC - PERU Calle del comercio 197 - San Borja, Lima - Perú (Citado el 16 de Junio del 2012). Disponible desde el URL: http:www.scielo. org.pe

2 MINISTERIO DE SALUD "Lineamientos para la dirección general de las personas adultas mayores" 2005 (Citado el 16 de Junio del 2012). Disponible desde el URL: http:www.upch.edu.pe/vrinve/ gerontologia/minsapdf/lineamientos.pdf2

3 Ministerio DE SALUd. Orientaciones técnicas para la Promoción de Alimentación y Nutrición Saludable. Disponible desde el URL: www.ins.gob.pe/gxpsites/agxppdwn.aspx? 2,4,315,O,S,0, $1733 \% 3 \mathrm{BS} \% 3 \mathrm{~B} 1 \% 3 \mathrm{~B} 134$

4 Institución EduCATIVA República DE Chile. Área de secretaria y admisión. Resoluciones de Docentes de la Dirección Regional de Educación de Ancash y Plana de Docentes. Casma, 2012.

5 Polit, D y Hungler, B. Investigación Científica en Ciencias de la Salud. Ed. Interamericana Mc. Graw-Hill; 4ta ed. México 2000.

6 HERnÁNDEZ R. Metodología de la Investigación. [Articulo en internet]. México: Editorial Mc Graw Hill; 2003. [Citado 2012 Jun. 16]. Disponible desde el URL:http://www.metabase.net/ docs/unibe/03624.html.

7 SchoenbaCh V. Diseños de estudio analítico. [Monografia en internet]. 2004. [Citado 2012 Jun. 16]. [44 paginas]. Disponible desde el URL: http://www.epidemiolog.net/es/endesarrollo/ DisenosDeEstudioAnaliticos.pdf. 
8 Canales, F y Alvarado, E. Metodología de la Investigación. Edit. Limusa, 20a. Reimpresión, México. 2004

9 Mendoza M. Estilo de vida y factores biosocioculturales de la persona adulta del AA. HH. Julio Méndez - Casma [Informe de Tesis para obtener el título profesional de Licenciada en Enfermería]. Chimbote: Universidad Católica Los Ángeles de Chimbote; 2009.

10 Regalado E, Osorio Y. Estilo de vida y factores biosocioculturales de la persona adulta - Carhuaz [Informe de tesis para optar el título de Licenciada en Enfermería]. Huaraz: Universidad Católica Los Ángeles de Chimbote; 2009.

11 FELIPE L. Estilo de vida y factores biosocioculturales del adulto mayor en el AA. HH. Villa Magisterial I y II etapa - Nuevo Chimbote [Informe de Tesis para obtener el título profesional de Licenciada en Enfermería]. Chimbote: Universidad Católica Los Ángeles de Chimbote; 2010.

12 FernandeZ, X y MendeZ, E. Estilos de vida y factores biológicos para la salud de las personas adultas del proyecto CRELLES. [Monografía de internet]. [Citado 2012 Jul 06]. [5 pantallas]. Disponible desde el URL: http://163.178.126.8/creles/cientif.htm

13 FERNÁNDEZ, L. Y REYES, S. Estilo de Vida y factores Biosocioculturales de la persona adulta A.H. Tres Estrellas [Tesis para optar el Título de Licenciado en Enfermería] Chimbote - Perú, 2009.

14 LaZARTE, C y MendeZ, A. Estilo de vida y factores biosocioculturales de la persona adulta del AA.HH Fraternidad. [Informe de tesis para optar el título de licenciatura en enfermería]. Chimbote: Universidad Católica Los Ángeles de Chimbote.2009.

15 Solis R. Chancahuaña V. Estlo de vida y factores biosocioculturales de la Persona Adulta del Centro Poblado Huallcor. [Tesis para optar el Titulo de licenciatura de enfermería] Huaraz: Universidad los Ángeles de Chimbote; 2010.

16 KoENIG, H. "Sociedades Bíblicas Unidas”, Sección: Reflexión e Inspiración: efecto de la religión en la salud, pag. 4: 07 de noviembre de 2007, EEUU. Disponible desde el URL: http://74.125.47.132/search?q=cache:R_ng8tc0A9sJ:labibliaweb.com/\%3Fp\%3D577+la + reli gion $+\mathrm{en}+\mathrm{la}+$ salud $+\mathrm{de}+\mathrm{las}+$ personas $\& \mathrm{~cd}=8 \& \mathrm{hl}=\mathrm{es} \& \mathrm{ct}=\mathrm{clnk} \& \mathrm{gl}=\mathrm{pe}$

17 CoRdero, M Y Silva, M . Estilo de vida y factores biosocioculturales de la persona adulta essalud - Chimbote. [Informe de tesis para optar el título de licenciatura en enfermería]. Casma : Universidad Católica Los Ángeles de Chimbote.2009

18 CRUZ, M. Y ReYES, F. Estilo de Vida y factores Biosocioculturales de la persona adulta del centro poblado menor de Ichoca - Huaraz [Tesis para optar el título de licenciado en enfermería] Huaraz Perú, 2010. 